\title{
Read-It: A Multi-modal Tangible Interface for Children Who Learn to Read
}

\author{
Ivo Weevers, Wouter Sluis, Claudia van Schijndel, Siska Fitrianie, \\ Lyuba Kolos-Mazuryk, and Jean-Bernard Martens \\ Design School of User System Interaction, University of Eindhoven, \\ Den Dolech 2, 5600 MB Eindhoven, The Netherlands \\ \{i.weevers, r.j.w.sluis, c.h.g.j.v.schijndel, s.fitrianie, \\ l.kolos-mazuryk\}@tm.tue.nl, j.b.o.s.martens@tue.nl
}

\begin{abstract}
Multi-modal tabletop applications offer excellent opportunities for enriching the education of young children. Read-It is an example of an interactive game with a multi-modal tangible interface that was designed to combine the advantages of current physical games and computer exercises. It is a novel approach for supporting children who learn to read. The first experimental evaluation has demonstrated that the Read-It approach is indeed promising and meets a priori expectations.
\end{abstract}

\section{Introduction}

In primary school, children aged five to seven years old start to learn how to read and write letters and words. The most frequently-used Dutch reading method, called Veilig Leren Lezen (VLL - Learning to Read Safely) [9], uses classroom activities, such as storytelling and verbal repetition, in combination with physical games and computer exercises, to practice reading. Currently, these latter exercises are assumed to be performed on a desktop computer. Since children at this age cannot read and write well, they mostly interact with the computer by means of the mouse. They, however, often experience difficulties while selecting, dragging and moving the cursor in a controlled way [15]. Although some argue against this by stating that these problems relate to the graphical user interface design and that children are more familiar with the mouse nowadays [10], interviews we carried out with primary school teachers, indicate that mouse handling problems occur frequently. Smets et al. [13] report that the interaction problems on a desktop computer are further increased by the fact that action (for example, the mouse movements) and perception (for example, the visual feedback on the screen) are spatially separated. The teachers whom we interviewed tend to confirm this point of view. Most importantly, however, desktop computers are based on a single-user/single-computer paradigm [14], and hence do not effectively support co-located, multi-user interaction. Scott et al. [12] have shown that children greatly enjoy technology that supports concurrent activities, and that forcing children to share one input device contributes to off-task behavior and boredom. Social interactions in a learning environment have also shown to lead to significant learning benefits [4,7]. Research by Inkpen et al. [5] demonstrates that 
children exhibit a significantly higher level of engagement and tend to be more active when working in parallel. Unfortunately, the face-to-face setup that is most often preferred for effective group interaction is prohibited by a desktop setting.

As indicated before, current reading methods use real card and board games to exercise reading skills within playful group activity. However, this game approach suffers from at least three problems. First, supervision by teachers is often lacking during such games, so that assistance and verification are not available. Second, the unique orientation of letters and words can cause perception problems. For example, children sitting on opposite sides can perceive the same letter as either a $/ \mathrm{d} /$ or a $/ \mathrm{p} /$. Third, most of these games are purely visually-oriented (for example, use only pictures and letters/words), so that the children mostly exercise the picture-word association, but not the letter-sound association.

It seems that the computer exercises and the tangible board games suffer from largely complementary problems. In our view, recent developments towards multimodal tangible interfaces offer the opportunity to combine the advantages of both methods, while avoiding the drawbacks. We developed the Read-It application: an interactive game that was inspired by the traditional card game "memory". Since it is a computer game, it can offer multi-modal feedback, consisting of written and spoken letters and words, in combination with pictures. Its interface allows for multiple simultaneously active interaction elements, and therefore allows for multiple simultaneous users, hence collaboration. Moreover, this tangible interface suits the motoric needs of the children.

\section{Previous Work}

Existing research projects have already explored many issues concerning the required technology, usability and possible applications of augmented tables (for example, Ishii and Ulmer [6], Fitzmaurice et al. [3], Rauterberg et al. [8], Aliakseyeu et al. [2]). Most of the presented applications have however only been tested with adult (usually, even highly professional) users. A much more challenging test for augmented table systems in our view is whether or not much less-experienced and trained subjects can also use and profit from such systems. Very young children as a user group, together with the identified problems of desktop computers and reading methods, influenced our choice for the design of an application with a clear educational goal.

There are only few existing projects in which tangible interfaces are used for creating collaborative learning experiences for children. One such prototype system is TICLE [11], which was designed for children that are "turned off by math and science" and shows promising results. Our prototype is different in at least two respects. First, our application is learning to read rather than solving mathematical puzzles. Second, we extend TICLE's design principles to a face-to-face setup and a coinciding action and perception space. In this way, we intend to establish more natural interaction between the children and between each child and the system.

Another project that we know off, but on which we have only limited information (see http://www.fit.fraunhofer.de/projekte/kiwi/), is the ZOOMlab. It was developed for the Vienna Children's Museum, and is intended to be used by up to 20 children 
simultaneously. It is implemented on the Assembly Table and focuses on assembly tasks, for example, on positioning multiple virtual objects on a large floor plan. The platform seems to be mostly intended for demonstrating the principle of tangible interaction. As far as we can deduce, no clear educational goals are pursued.

\section{Read-It Design Rationale}

Since the focus of our project is not on technological development, but on designing and testing an educational application, we have chosen to implement the Read-It prototype on an existing augmented tabletop environment, the Visual Interaction Platform (VIP) [2]. The platform consists of a computer, one beamer, an infrared light source, an infrared-sensitive camera, and a table with a reflective surface. The beamer projects the computer screen output onto the horizontal table. The infrared light source illuminates the action-perception space on the table. The light is reflected towards the infrared-sensitive camera by means of retro-reflecting tags. These tags are attached to physical bricks that are positioned on the table. The computer runs computer-vision software to analyze the images from the camera. The software output consists of tag - and thus brick - positions, orientations and unique tag identifications. Each brick has a unique tag, which pattern is defined by a number and the position of non-reflecting holes in the tag.

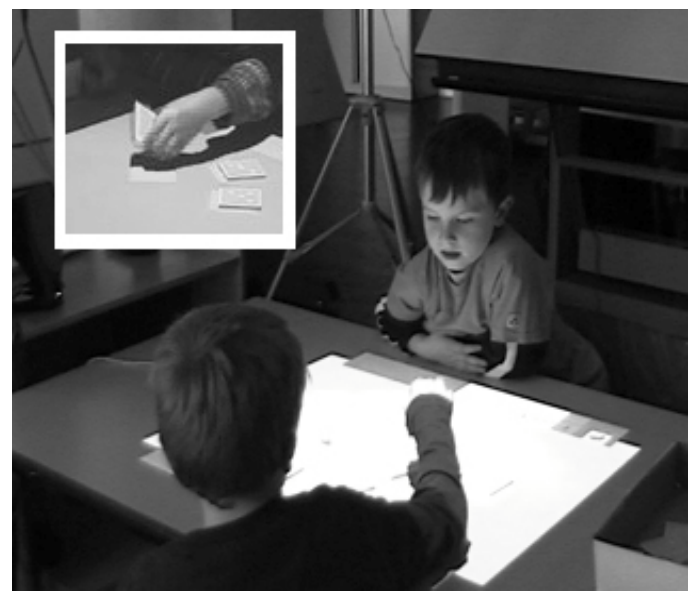

Fig. 1. Two children playing the Read-It game. Top-left: Flipping a tangible brick.

Our application is inspired by the traditional game of "memory". It uses twenty tangible bricks, each associated with a virtual memory card. The virtual cards are projected on top of the physical bricks. The sides of the bricks that are facing upwards when the game is started are associated with the blind sides of the virtual memory cards. The invisible sides are associated with the pictures (and words). By flipping a brick, the corresponding virtual memory card is flipped and the system shows or hides the card's picture. Figure 1 shows a child flipping a brick and thus a virtual card. 


\subsection{The Game}

The goal of the Read-It game is to find all matching picture pairs. Two pictures match when their related words have either identical first, middle or last letters (depending on the game type). For example, the Dutch words "vis" (fish) and "vos" (fox) match in their first letter, while "vis" and "pen" (pen) do not. Children play in couples and they are encouraged to collaborate. When the children think two pictures match they have to press a large button next to the projection area. If their decision is correct, they receive a carrot picture as a reward. If their decision is incorrect, the virtual cards show a red border and the children have to turn them back over before continuing. The game ends when the children have found all matching pairs. A large animated carrot is the final reward.

\subsection{Multi-modality}

Training through different senses benefits the children's reading skills. Especially in early reading education, children need to acquire knowledge about the correct grapheme (the graphical character $/ \mathrm{v} /$ ) to phoneme (the sound [v]) mapping. Read-It offers the children the opportunity to practice this mapping by offering both audio and video feedback. When the children reveal a new picture by flipping a brick, the ReadIt application displays and spells the associated letters and word visually and auditory, in a synchronized way.

\subsection{The Orientation Problem}

Reading displayed letters from opposite sides of a table raises an orientation problem. For example, one child might see a /d/, while the child sitting on the opposite site will perceive the same letter as a $/ \mathrm{p} /$. Such a situation is especially troublesome for novice readers and is hence to be avoided. We solved the problem by separating the projection area into one shared and two personal workspaces. Figure 2 shows the image being projected onto the table by the Read-It game. The shared workspace holds the virtual cards and displays only pictures. When a new picture is revealed, the system displays a smaller version of the same picture, together with the corresponding spelled word, in each personal workspace. This prevents that the children have to shift their attention from the personal to the shared workspace while reading a word. We expect this to benefit the picture-word mapping.

\subsection{Movement of Focus}

The focus of attention is purposely shifted around during the course of the game, by involving different areas when flipping the cards, by spelling the words both visually and auditory, and by the need to press the confirmation button. Acuff [1] has pointed out that children like to be busy and entertained and react positively to frequent stimulation. With Read-It, children do not need to keep still and concentrate continuously on the same thing. We expect this to favor their engagement. 


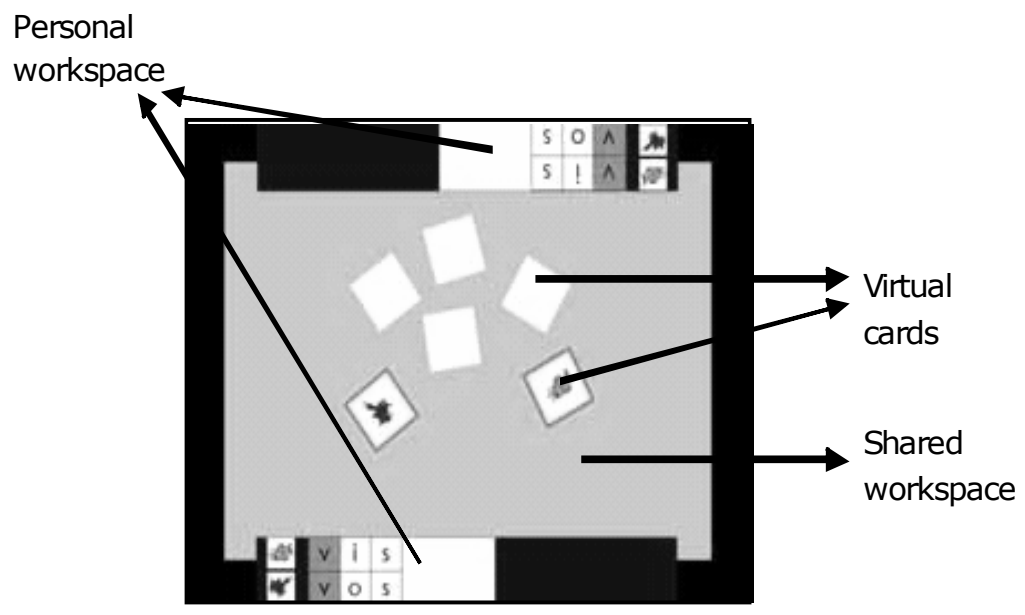

Fig. 2. The Read-It interface with its shared and personal workspaces.

\subsection{Tangible Objects}

Bricks avoid two of the above-mentioned desktop problems. First, they match the children's motoric skills better than a mouse. Second, the bricks on the augmented table realize a combined action-perception space. Another advantage, as indicated by some of the teachers, is that bricks agree with many children's preference towards brick toys, such as LEGO. In order to obtain reliable brick recognition, the brick size was fixed at $5.5 \times 5.5 \mathrm{~cm}$. We tested bricks with varying heights $(2,6,8,16,18$, and 22 $\mathrm{mm}$ ) with 37 five-to-seven-year old children to select a brick height that suited the children's motoric skills and preference. Furthermore, we tested bricks with and without a notch along the sides. Such a notch makes it potentially easier to grasp and turn the brick. On average, the brick with a height of $6 \mathrm{~mm}$ and with a notch was preferred.

\subsection{Collaboration}

Unlike desktop games, the Read-It application is a cooperative game that promotes natural face-to-face communication. Since the game uses twenty bricks that are scattered around the table, collaboration benefits the children in reaching their goal. They can collaborate both mentally, in order to remember the locations of the pictures, and physically, in order to reach the bricks to be flipped and to press the confirmation button. This collaboration is further strengthened by the fact that the Read-It application supports parallel activity by the children through simultaneously active interaction elements. We expect that education styles for children that support such daily social interactions result in learning benefits [4,7]. 


\subsection{Support for Learning}

Teachers use reading games in class to strengthen the recall and rehearsal of words and letters. Both learning mechanisms are essential in the process of learning how to read and are supported by Read-It. Children flip the same card multiple times, which makes them read the corresponding letters and hear the related sounds more than once. Although children will find some matching cards fairly fast (by shear luck), the game holds multiple instances of each letter, so that all letters/sounds are rehearsed.

\section{Experimental Evaluation}

Before setting up a large longitudinal study to find out about Read-It's effects on reading skills, we needed to evaluate whether or not our system meets first requirements. We designed an experiment to evaluate whether or not five-to-sevenyear-old children are able to understand and work with the augmented application. We also wanted to find out whether or not face-to-face collaboration within the couples is established, which we consider to be an indication for achieved social communication.

\subsection{Experimental Design}

The experiment involved 15 children between five and seven years old. Twelve of them formed 6 pairs. Three children were paired with a member of the research theme. The observations for these three children were only used for analyzing how they handled augmented reality, not for analyzing collaborative behavior.

All children knew the traditional memory game, which has the goal of competing against each other. However, with Read-It the children are encouraged to collaborate. Therefore, in order to get acquainted with the new game rules, the couples started by playing a memory game with Read-It game rules using real cards. Next, in order to become familiarized with the augmented environment of the Read-It system, and in order to adjust to the laboratory setting, the children played an introductory Read-It game with three card pairs. Afterwards, the children played a full game with ten card pairs. Finally, the children had to explain to their parents with another three-pairgame what they had experienced. The parents could not observe their children during the previous sessions. The sessions were taped (two camera's recording each child from the front and one camera recording the children's action space). We used the tapes to observe peculiarities and to analyze the children's behavior with the coding schemes explained below.

\subsection{Results}

Augmented Reality. We used the number of explorative acts to quantify the children's understanding of the concept of augmented reality, for example, real bricks being combined with virtual cards. Children show an explorative act when they (1) 
pick up a card and look at it without flipping it, (2) peak under a card before turning it, and (3) express that they understand the match of the brick with the projection of the virtual card. We divided the explorative acts into four timeslots across each game. The total time, which is measured from flipping the first card to all cards facing downwards again after successful matching of all cards, varied. It took the couples on average 23 turns to finish the ten-pairs-game. Figure 3 plots the average number of explorative acts across all 15 children as a function of game quarter. It demonstrates that the explorative behavior of the children decreases fast. It is already reduced to $50 \%$ towards the end of the relatively short game.

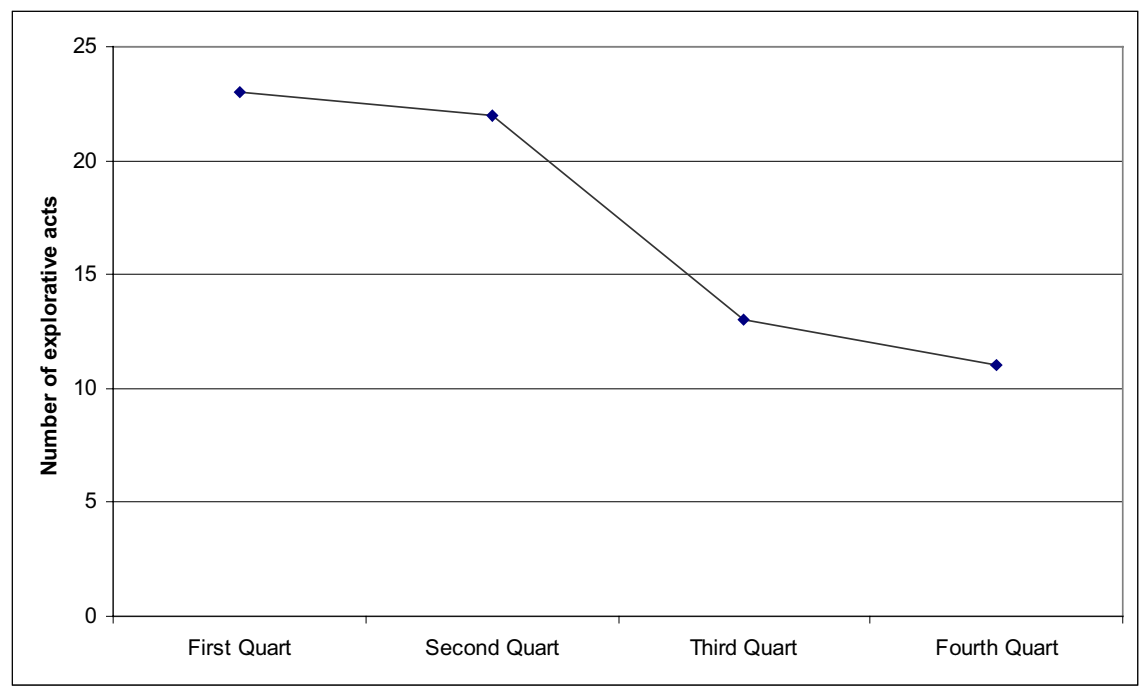

Fig. 3. Total number of explorative acts of the children as a function of the phase within the game.

Collaboration. We used four indicators for collaborative acts: (1) discussion about which card to flip, (2) discussion about which letter to match with, (3) making a gesture to direct the other child's attention to a specific card, and (4) looking at each other for confirmation. During the game we observed an average of 5.5 collaborative acts per couple. Large differences, however, occurred between couples, probably due to differences in dominance between the children within a couple.

\section{Discussion}

We think that the Read-It game will support the process of learning how to read in the long run. The pilot study confirms that the children are able to grasp and use the major system concepts, such as augmented reality and the multimodal environment. All children finished the game and their exploratory behavior reduced quickly. Also, seven out of fifteen children read aloud along with the visual and auditory spelling by the system. The study also demonstrated that the children enjoyed playing and 
learning with the applied technology. One could argue that the appearance of personal workspaces triggers the players towards an individualistic game strategy. However, a certain degree of collaboration between the children was clearly established.

\section{Conclusion}

We presented Read-It, a novel approach for supporting young children in the process of learning how to read. Read-It overcomes some identified problems of current desktop exercises and board games that support the reading method VLL. The game uses tangible bricks on a tabletop for improved interaction and applies different mechanisms - collaboration, recall and rehearsal - to support the learning process. Read-It was designed for enriching the process of developing reading skills. As such, the contribution of the system to the children's reading experience is a critical aspect. Understanding the true effect on reading skills would require a longitudinal study. Before conducting such a study, we performed a short pilot experiment to evaluate: (1) whether or not children of five-to-seven-years old are able to work with the concept of combined real and virtual elements, and (2) whether or not collaborative behavior was manifested. The conclusion on both aspects was positive, so that we can argue that we have indeed created a rich learning environment that encourages children. Altogether, the presented design and evaluation provide a good foundation for further research.

\section{References}

1. Acuff, D. S. What Kids Buy and Why: The Psychology of Marketing to Kids. Free Press, Detroit (1997).

2. Aliakseyeu D., Martens J.B., Subramanian S., Vroubel M. and Wesselink W, "Visual Interaction Platform", Proc. Interact 2001, Tokyo (2001) 232-239.

3. FitzMaurice, G.W., Ishii, H. \& Buxton, W., Bricks: Laying the Foundation for Graspable User Interfaces, Proc. Of CHI '95, ACM Press (1995) 170-177.

4. Hymel S., Zinck B., Ditner E., Cooperation versus Competition in the Classroom. Exceptionality Education Canada (1993) 3:1-2, 103-128.

5. Inkpen, K.M., Ho-Ching, W., Kuederle, O., Scott, S.D., Shoemaker, G.B.D. "This is fun! We're all best friends and we're all playing.": Supporting children's synchronous collaboration. Proc. of CSCL '99, ACM Press (1999) 252-259.

6. Ishii, H., and B. Ulmer. Tangible Bits: Towards Seamless Interfaces between People, Bits, and Atoms. Proc. CHI '97, ACM Press (1997) 234-241.

7. Johnson D.W., Maruyana G., Jonhson R.T., Effects of Cooperative, Competitive and Individualistic Goal Structures on Achievement: A Meta-Analysis, Psychology Bulletin, (1981) 89(1), 47-62.

8. Rauterberg, M., Fjeld, M., Krueger, H., Bichsel, M., Leonhardt, U \& Meier, M., BUILDIT: a video-based interaction technique of a planning tool for construction and design, Proc. Work with Display Units (WWDU) '97, (1997) 175-176.

9. Reitsma, P. \& Verhoeven, L., Acquisition of Reading Dutch, Foris Publications, Dordrecht, the Netherlands (1990). 
10. Romeo G.I., Edwards S., McNamara S., Walker I., \& Ziguras C., Investigating the Potential of Touchscreen Technology in Early Childhood Education, Proc. AARE '01 (2001).

11. Scarlatos L.L., TICLE: Using Multimedia Multimodal Guidance to Enhance Learning, Information Sciences 140 (2002) 85-103.

12. Scott S.D., Mandryk R.L., Inkpen K.M., Understanding children's collaborative interactions in shared environments, Journal of Computer Assisted Learning (2003) 19, 220-228.

13. Smets, G.J.F., Stappers, P.J., Overbeeke, K.J. and Van der Mast, C., Designing in virtual reality: Perception-action coupling and affordances. In: Carr, K., and England, R. (eds.), Simulated and Virtual Realities. Elements of Perception, Taylor \& Francis, London (1995) 189-208.

14. Stewart J., Bederson B.B., Druin A, Single Display Groupware: A Model for Co-present Collaboration. Proc. CHI '99, ACM Press (1999) 286-293.

15. Strommen E. Children's Use of Mouse-Based Interfaces to Control Virtual Travel, Proc. SIGCHI on Human Factors in Computing Systems '94, ACM Press (1994) 405-410. 